Commentary on The Unfolding Case Formulation: The Interplay of Description and Inference (Eells), and Using Systematic Case Studies to Investigate Therapist Responsiveness: Examples from a Case Series of PTSD Treatments (Edwards)

\title{
Evaluating Case Formulation Decision-Making and Therapist Responsiveness: A Perspective from the Area of Behavioral Assessment and Case Formulation
}

\author{
WILLIAM H. O'BRIEN ${ }^{\text {a,b }}$ \\ ${ }^{a}$ Department of Psychology, Bowling Green State University \\ b Correspondence concerning this article should be addressed to William H. O'Brien, Department of Psychology \\ Bowling Green State University, Bowling Green, Ohio 43403. \\ Email: wobrien@bgsu.edu
}

\begin{abstract}
In this paper two research studies by Eells (2010) and Edwards (2010) evaluating case formulation and therapist responsiveness are summarized and critically evaluated. The central themes abstracted from these studies are subsequently articulated and integrated with existing research on the philosophical and theoretical competencies, clinical competencies, and quantitative competencies needed for effective case formulation. More specifically, I introduce and evaluate the work of Edwards and Eells in terms of a behavioral model of case formulation that is based on four general principles: functionalism; empiricism, cognitive-behavioral learning theory; and a multivariate perspective that stresses the importance of collecting multimodal and multimethod assessment data as the basis of a case formulation.
\end{abstract}

Key words: case formulation; therapist responsiveness; behavioral assessment; functional analysis; quantitative analysis; case studies

\section{OVERVIEW}

Two papers, respectively authored by Eells (2010) and Edwards (2010), explore important aspects of psychotherapy: case formulation competencies and clinician responsiveness within the context of manualized treatments. Additionally, each paper reflects on the unique contribution of idiographic/qualitative methods in furthering our understanding of these complex phenomena.

In this paper, a summary and critical review of each paper is provided. Subsequent to this, the common themes addressed by Eells and Edwards are discussed more fully. Finally, additional case formulation competencies are presented for consideration along with an argument 
that a core expert clinical competency is being able to incorporate quantitative reasoning with qualitative case formulation.

\section{EELLS’ PAPER: SUMMARY AND COMMENTARY}

\section{Summary}

Noting that single subject research methods can complement nomothetic approaches, Eells (2010) sets out to evaluate the extent to which these methods capture unique and common information about clinical judgment among novice practitioners (graduate students in psychology), experienced clinicians (therapists with approximately 20 years of experience), and expert clinicians (therapists with 20 years of experience, publications relevant to case formulation, and extensive supervisory experience). To accomplish this goal, Eells developed six vignettes that presented different disorders (anxiety disorder, affective disorder, and borderline personality disorder) and two different levels of "prototypicality" (low, high). The clinicians read the vignettes and generated verbal accounts of their case formulation and treatment approach. The verbal accounts were then audio-recorded and coded for comprehensiveness, coherence, precision of language, the degree of elaboration of treatment plans, the complexity of information, the link between the formulation and treatment plan, and the degree to which the formulation development appeared to be systematic. Additionally, Eells evaluated the extent to which the therapists used forward reasoning (i.e., verbalizing a description of a behavior and then an inference about the causes of the behavior) relative to backwards reasoning (i.e., verbalizing an inference about a behavior and then a description).

At the group level, Eells' data indicated that the expert therapists generated "higher quality" (an aggregate of the various ratings) case formulations relative to experienced therapists and novices. The experts also talked more-they generated more "descriptive, diagnostic, inferential, and treatment planning information than nonexperts." Finally, there was some evidence that the experts produced more descriptive-inference statements relative to the nonexpert experienced therapists.

After obtaining the aforementioned nomothetic results, Eells then pursues an idiographic approach. To achieve this, the two highest quality case formulations (one cognitive-behavioral, one psychodynamic) for the most difficult vignette (borderline symptoms with low prototypicality) were selected and compared with "more ordinary formulations" from experienced therapists. A cumulative frequency graph was then generated for each therapist. The cumulative frequency graphs charted the occurrence of descriptive statements, inference statements, and treatment-related statements. To evaluate these data, Eells focuses on the extent to which the lines converge or diverge across the seven-minute case formulation narrative. Augmenting these graphed data, Eells presents sample text from the clinicians to contextualize the decision-making process and attendant coding scheme.

Based on idiographic data, the finding of descriptive-inference processes was again observed. The analysis illustrated how the nonexpert clinicians tended to generate inductive comments that, on many occasions, had little correspondence with information in the vignette. 
Conversely, the experts tended to use more precise language and proceed in a more systematic manner, first articulating descriptions of problems and then offering interpretations and inferences.

In conclusion, Eells suggests that the "aggregate then analyze” (nomothetic) and "analyze then aggregate” (idiographic) approaches are complementary with the former being more akin to a randomized clinical trial and the latter being a "contextually rich and empirically supported clinical case study containing both qualitative and quantitative information.” More specifically it is argued that while both methods tended to produce findings indicating differences in approach between nonexperts and experts, the idiographic data was more able to illustrate differences in the decision-making processes among clinicians. Finally, Eells presents a conceptual model of "case formulation guided therapy" in which the clinician proceeds, in an iterative and recursive fashion, through a series of steps that involve gathering information, formulating, treating, and terminating. Further, within the formulation stage, the clinician proceeds through four more linear steps that include: create problem list, diagnose, develop explanatory hypotheses, and plan treatment.

\section{Commentary}

Eells is addressing an interesting and difficult to evaluate aspect of clinical work-the manner in which clinicians evaluate client information in order to render a case formulation and treatment plan. Additionally, Eells compares and contrasts information derived from nomothetic and idiographic approaches suggesting that the latter provides a more contextualized understanding of the decisional processes used by clinicians in the case formulation process.

The construction of the research question, methods, and conclusions offered by Eells are reasonable. The use of cumulative graphs to evaluate the narratives offered by the clinicians is an interesting use of this methodology and provides valid insights into the relationships among the three coded variables. And, it seems intuitive to make the argument that expert clinicians are more able to generate comprehensive and higher quality case formulations than nonexperts. Finally, the verbatim narratives do provide excellent illustrations of the information summarized by the graphs.

There are, however, some concerns to consider in the interpretation of findings offered by Eells. The most salient are related to construct validity and ecological validity. A principal aim of the project is to capture the decisional processes that are used by clinicians in assessment and case formulation. As noted by ourselves and many others (cf. Haynes \& O’Brien, 2000; Garb, 2005), clinical assessment and case formulation is an interactive process involving reciprocal causal relationships — client behavior affects therapist behavior, and vice versa. The process is also dynamic (i.e., changing across time); multimodal (i.e., involving multiple response modes including cognition, affect, observable behavior, physiological reactions, and verbal reports); and context-bound (i.e., the behaviors are influenced by immediate and distal causal factors affecting client behavior). Thus, Eells' measures of decision-making and case formulations derived from codes abstracted from clinician narratives as they reflected on the reading of a vignette about a fictitious client very likely under-specify the construct of interest (i.e., only captures a small 
component of clinical decision-making). Additionally, the complexity decision-making in realworld contexts is not well-captured by this methodology.

A second construct validity concern is related to the very idea of "quality." In this particular paper, quality is represented by the comprehensiveness of narrative along with a number of other codes applied to clinician narratives. In a sense, the authors may be measuring facility with narrative construction rather than adequacy of case formulation. And, it stands to reason that experts who have extensive experience in supervision and case formulation research would be more able to verbalize their assessment decisions. However, this capacity for verbalizing thoughts about assessment information and case formulation is not equivalent to an assessment of the validity of case formulation. For example, there is no clear indication of what the "correct" formulation and treatments are for these clients. In absence of having this information, there is no way to determine whether the decisions offered by the clinicians are, in fact, more or less adequate.

\section{EDWARDS' PAPER: SUMMARY AND COMMENTARY}

\section{Summary}

Edwards (2010) also aims to explore an important and complex phenomenon in psychotherapy: therapist responsiveness. Edwards notes that responsiveness is a critically important contributor to therapy outcomes and that it can range from micro interactions occurring from moment-to-moment within a single session to long-term interactions that can span months or even years. Edwards also makes the point that opportunities for therapist responsiveness vary along a dimension that ranges from minimally interactive interventions (e.g., self-help books) to very interactive, "formulation driven" interventions (e.g., the clinician generates an individualized case formulation and intervention), with interventions affording moderate levels of interaction (e.g., assisted self-help, specific protocols for specific problems) falling in the middle.

According to Edwards, therapist responsiveness can be partitioned into two broad domains. One is described as an area of general competencies where the clinician must be able to provide specific intervention components while simultaneously being responsive to the "nonspecific" elements of therapy (e.g., monitoring engagement). The second area is labeled metacompetencies. In this domain, the clinician must be responsive to another level of client needs which subsumes a range of immediate and distal contextual factors (e.g., the clinicianclient relationship quality, sociodemographic factors, family factors, etc. ).

Because most empirically-supported interventions use detailed manuals and emphasize the evaluation of client outcomes using group-based methods, little is known about the extent to which therapist responsiveness contributes to outcomes. Edwards goes on to make the point that the use of qualitative case-study methods can provide insight into therapist responsiveness. To illustrate, Edwards presents narratives from six clients who were evaluated and treated for posttraumatic stress disorder. The procedures used to evaluate and treat these clients are examined in relation to an "evidence-based responsive treatment planning" model that involves 
complex decisional processes organized into three levels: crisis intervention and stabilization; promoting engagement with treatment; and selection, sequencing, and timing of active treatment components. In conclusion, Edwards argues that effective manual-driven therapy requires flexibility. That is, the therapist must actively and carefully monitor client reactions as well as a host of contextual factors. In turn, this information must be used to make adjustments and modifications of the treatment protocol as therapy unfolds.

\section{Commentary}

The Edwards paper presents a compelling argument for conducting systematic research into therapist responsiveness within the context of manualized treatments. A number of researchers have pointed out that therapy process-outcome relationships in cognitive-behavioral therapy have been under-studied (e.g., Webb, DeRubeis, \& Barber, 2010; O'Brien, Korchynsky, Fabrizio, McGrath, \& Swank, 1999; Tsai, Kohlenberg, Kanter, Kohlenberg, Follette, \& Callighan, 2008). Interestingly, therapist deviation from protocol is viewed as a threat to study integrity in a randomized clinical trial. Yet, as Edwards notes, in real-world clinical contexts the same behaviors are commonplace and can be thought of as therapist responsiveness.

Edwards' presentation of narratives on how social support can be used to aid in the protocol-based treatment of posttraumatic stress disorder provides a nice illustration of the integration of responsiveness and protocol adherence. The themes derived from these case studies also provide insight into how a supervisor can help supervisees acquire skills in responsiveness.

One of the difficult points in Edwards paper is the argument that positivism and empiricism impede understanding of therapist-client interactions, when he speaks about "blind adherence to positivist principles which exclude from science any findings not derived from quantification and statistical hypothesis testing" (p. 258). While it is the case that adopting an empirical and positivistic stance constrains some aspects of therapist-client relationship research, many researchers have been able to use these approaches very effectively. Additionally, these empirically-based studies have yielded very rich data sets that provided important insights into the process-outcome relationships in therapy (for an excellent example of therapist-client research that uses rigorous empirical methods see Tsai et al., 2007).

\section{INTEGRATION AND EXTENSION OF ISSUES ADDRESSED BY EELLS AND EDWARDS}

The Eells and Edwards papers are exploring two interrelated themes that merit further discussion: (a) clinician decision-making related to case formulation and treatment planning, and (b) the relative merits of qualitative idiographic research methods relative to quantitative idiographic research and nomothetic research. These important issues are discussed more fully in the following sections. More specifically, below I present a behavioral model of case formulation that is based on four general principles: functionalism; empiricism, cognitive-behavioral learning theory; and a multivariate perspective that stresses the importance of collecting multimodal and multimethod assessment data as the basis of a case formulation. In this presentation I will focus 
on case formulation and treatment decision-making competencies that emerge from the behavioral model that were not directly examined by the Eells' and Edwards' papers and which, I suggest, would enhance the approaches they lay out.

\section{Clinician Decision Making and Case Formulation}

Within the first few moments of a typical encounter between a clinician and a client, literally thousands of bits of information are available for evaluation. This information will take the form of client reports of behavior problems, the causes of those behavior problems, background factors and historical events, and myriad other statements (some of which have little bearing upon the presenting problems). Beyond the semantic content of verbal reports, there will also be many additional sources of information that the clinician can observe such as: paralinguistic features of speech (voice rate, volume, eye contact, etc.), emotional state, cognitive functioning (e.g., attention and concentration skills, memory biases), and physiological responses. Importantly, the client will also be evaluating the clinician's behavior and responses to what he or she says so that within minutes a reciprocal causal interaction is unfolding. Approximately one hour later, at the conclusion of this initial meeting between clinician and client, a vast amount of information has been exchanged. Additionally, at this point, the clinician will oftentimes have generated a preliminary case formulation that will be quite immutable.

Many important questions about this process of case formulation have been asked in the assessment research. Some of the more central questions are: (a) How does a clinician sort through all of this information, (b) how does a clinician determine what information is important or irrelevant, (c) how does a clinician construct a case formulation from this information, and (d) how valid are case formulations?

Questions (a) and (b) have been more extensively studied, and we therefore have a better grasp of how clinicians attend to, encode, and recall information presented during assessments. Questions (c) and (d) have been less completely studied and are simultaneously very difficult to research. Thus, we have a very incomplete understanding of the construction process in case formulation and the validity of case formulations.

Eells and Edwards identified interesting and important aspects of decision-making within the context of case formulation. However, there are a number of additional competencies that are required to gain a better understanding of the case formulation process. These competencies also address the four questions outlined above. In the following sections, these additional key clinical competencies are discussed. Importantly, some of these competencies directly address the qualitative-quantitative distinction presented by Eells and Edwards.

\section{$\underline{\text { Philosophical and Theoretical Competencies in Case Formulation }}$}

Understanding the essential philosophical and theoretical characteristics of a particular psychological paradigm is critical for assessment, clinical decision-making, case formulation, and treatment implementation. An important assumption that cuts across paradigms is functionalism (Haynes \& O’Brien, 2000). Functionalism is a philosophical and theoretical 
approach which posits that behavior problems exhibited by clients occur as a function of complex causal influences that can be partitioned into intrapersonal events (e.g., physiological states, cognitive experiences, learning history, etc.); social environmental events (e.g., interpersonal interactions); and nonsocial environmental events (e.g., settings, time of day, etc.).

From a functional perspective, the essential unit of analysis is the relationship between behaviors and the contexts within which they occur. Thus, behaviors cannot be isolated from the environment and evaluated for form and content. Instead, the focus is on how and why target behaviors occur within specific and well-defined intrapersonal, social environmental, and nonsocial environmental settings.

A solid understanding of empiricism is another critical element of contemporary assessment and case formulation. This characteristic is important because it provides support for the use of well-developed and minimally inferential operational definitions of behaviors and contextual variables in assessment and case formulation. Further, there is a preference for the use of measures that permit quantification of behaviors, contextual variables, and the relationships among them. The empirical position does not negate the utility of qualitative approaches. As noted by Eells, the expert therapists carefully and frequently generate descriptions of behavior prior to generating inferences. This is consistent with an empirical approach.

A third characteristic of current assessment and case conceptualization approaches is a reliance on psychological theories to explain how and why behaviors are acquired, maintained, and changed. The crucial learning theories required for cognitive-behavioral approaches are classical conditioning, operant conditioning, and social learning theory. It is also becoming apparent that functional contextualism, an extension of functionalism, is emerging as an important element of a cognitive-behavioral approach to human behavior (Hayes, Luoma, Bond, Masuda, Lillis, 2006). For psychodynamic approaches, the critical theories may be derived from intrapsychic dynamics, defense mechanisms, transference, and object relations (Huprich, 2009).

Finally, current assessment and case conceptualization approaches espouse a multivariate perspective in which it is presumed that behaviors and contexts are comprised of many elements. Further, there are myriad ways that these behavioral and contextual elements can relate to one another. Some relationships will be correlational and some will be causal. Thus, assessments should use multimodal and multimethod measurement with an aim of identifying the more important relationships among behavioral and contextual elements.

\section{Clinical Competencies in Case Formulation}

The primary goal of assessment is to improve clinical decision making by obtaining reliable and valid information about the form and function of target behaviors. This primary goal is achieved when two subordinate goals are met: (a) objective measurement of target behaviors, and (b) identification of contextual factors that exert important influences on target behaviors. To achieve these two goals, therapists must have basic competencies in processes used to generate operational definitions of target behaviors and contextual factors. Additionally, 
strategies for collecting empirical data about relationships among target behaviors and contextual factors must be developed and implemented. Finally, after data collection, effective evaluation procedures must be used to measure the magnitude of relationships between target behaviors and contextual factors. In the following sections, these basic assessment competencies are reviewed.

Generating descriptions of behavior and context. A primary goal of assessment is to accurately measure target behaviors. To accomplish this, the therapist must determine which behaviors are important and how they should be measured. Operational definitions are used to capture the specific and observable elements of target behaviors. When developing an operational definition, the therapist should emphasize content validity. And, in line with the multidimensional assumption, it is expected target behaviors will need to be operationalized in a number of different ways.

In order to simplify the operationalizations, Stephen Haynes and I (2000) recommend that behaviors be divided into three modes of responding: verbal-cognitive, physiologicalaffective, and overt-motor (Haynes \& O’Brien 2000). The verbal-cognitive mode includes spoken words and cognitive experiences (self-statements, beliefs, attitudes). The physiologicalaffective mode includes physiological responses, sensations, and emotional states. Finally, the overt-motor mode includes observable motor responses.

Once a behavior has been operationally defined in terms of modes, the behavior therapist must determine which response parameters are most relevant. The most commonly used measurement parameters of target behaviors are frequency, duration, and intensity. In addition to determining which parameter of measurement will be used to quantify a target behavior, the therapist will need to measure the relative importance of a target behavior if more than one is described by the client (which is most often the case).

Subsequent to generating operational definitions of behaviors, the therapist needs to generate operational definitions of contextual factors. Contextual factors are environmental events that exert important effects on the target behaviors. Contextual factors can be sorted into two broad categories: social/interpersonal events and nonsocial/environmental events.

Social/interpersonal factors include interactions with other living beings-most typically people and groups of people. Nonsocial/environmental factors include interactions with the "nonliving” elements in the environment. Examples of nonsocial/environmental factors include the structure of the built environment, noise levels, temperature, work schedule, work load, and the like. These nonsocial/environmental causal factors tend to be understudied but can exert a significant impact on behavior.

The measurement parameters of contextual factors are similar to those used with target behaviors. Specifically, there is an emphasis on measurement frequency, intensity, and/or duration of contextual factor occurrence. Additionally, it is helpful to rate the clinical utility of a contextual factor. The clinical utility rating is an estimate of the extent to which a particular contextual factor can be modified for the purposes of creating a change in behaviors. For example, some important contextual factors can be unmodifiable (e.g., temperature, season, work characteristics, presence of a partner who is unwilling to participate in therapy, etc.). While 
these important contextual factors should be noted and understood as having an impact on target behaviors in the case conceptualization, they are de-emphasized in interventions because of their immutability.

In summary, the topographical analysis of target behaviors involves generating operational definitions of cognitive, affective, and motor responses. Additionally, importance ratings of the target behaviors are obtained. The topographical analysis of contextual factors involves generating operational definitions of intrapersonal, interpersonal, and environmental events that exert important causal effects on target behaviors. These contextual factors are then rated in terms of modifiability or clinical utility.

Estimating relationships among target behaviors and contextual factors. Once the topographical analyses of target behaviors and contextual factors have been completed, the therapist must evaluate interrelationships among target behaviors and contextual factors. In most cases the emphasis is on identifying and evaluating a subset of all possible relationships — those that have causal properties. Reliable covariation between a target behavior and contextual factor is a critical index of possible causality. However, covariation alone does not imply causality. Causal relationships should meet three criteria: (a) temporal order, i.e., the changes in the causal variable should precede effects on the target behavior, (b) a theoretical explanation for the relationship (based on logic and learning theory), and (c) the exclusion of plausible alternative explanations for the observed relationship.

Three basic methods can be used to identify causal relationships, and a therapist should have competencies in using all three methods. The marker-variable strategy is the most commonly used method of inferring causality. A marker variable is an easily obtained measure that denotes the presence of a causal relationship. Client interview responses are typically used as marker variables. For example, a client statement that his or her migraine headaches is almost always "triggered" by stress is taken as an index of a potentially strong causal relationship between stress and headaches in daily life. Marker variables can also be derived from selfreport inventories specifically designed to identify functional relationships, structured interviews, and structured therapist-client interactions.

A second procedure commonly used to obtain information about causal relationships is systematic observation of naturally occurring target behavioral-causal variable interactions. Most commonly, assessors use client self-monitoring to evaluate causal relationships in this way. For example, a client may be instructed to record the daily levels of stress and headache activity to determine whether there is a causal relationship between these two variables. An alternative strategy is to have trained observers record target behavior-causal variable interactions. An example of this approach would be to have observers record the occurrence of a child's off-task behavior and subsequent social reinforcement by peers in a class room setting.

The third method that can be used to identify casual relationships is experimental manipulation. Experimental manipulations involve systematically modifying a causal variable while observing consequent changes in target behavior. These manipulations can be conducted in the natural environment, in analog settings (e.g., Iwata, Smith, \& Michael, 2000), in 
Evaluating Case Formulation Decision-Making and Therapist Responsiveness:

A Perspective from the Area of Behavioral Assessment and Case Formulation

W.H. O'Brien

Pragmatic Case Studies in Psychotherapy, http://pcsp.libraries.rutgers.edu

Volume 6, Module 4, Article 6, pp. 293-306, 12-22-10 [copyright by author]

psychophysiological laboratory settings (e.g., O’Brien \& Haynes, 1995), and during assessment and therapy sessions (Tsai, et al. 2008).

It has been recommended that a clinician's use of different methods at different points in the case conceptualization. In the early stages, the assessor can rely on marker variables so that many potential causal relationships can be identified. Later, after additional information has been collected on behavior-causal variable interactions, the clinician can select a subset of important causal relationships and subject them to more intensive scrutiny using self-monitoring, observation, and/or systematic manipulation.

Generating a clinical case model. Once target behaviors and contextual factors have been operationalized, a working model of interrelationships among these variables needs to be developed. This conceptual model of interrelationships between behaviors and contextual factors has also been labeled the "functional analysis," which Haynes and I have more formally defined as "the identification of important, controllable, causal functional relationships applicable to a specified set of target behaviors for an individual client" (Haynes \& O'Brien, 1990, p. 654).

The clinical case model is a core methodology in assessment because it summarizes and integrates behavior-context interactions and addresses the onset, maintenance, and modification of behavior occurrence. We have recommended that therapists generate functional-analytic causal models to depict functional analyses. Such models are vector diagrams that graphically depict the interrelationships among target behaviors and contextual factors for an individual client (see Haynes \& O’Brien, 2000; and Haynes, O’Brien, \& Kaholokula, in press).

\section{Quantitative Competencies in Case Formulation}

Once a case formulation or clinical case model has been developed, the therapist will need to estimate the magnitude of relationships among key behaviors and causal variables. Most often, therapists rely on intuitive decision-making for this element of the case conceptualization. Unfortunately, there are many studies that have consistently demonstrated that therapists simply cannot reliably or accurately estimate causal relationships using intuition alone (Chapman \& Chapman, 1969; Dollinger, Greening, \& Radtke, 2001; Gilovich, Griffin, \& Kahneman, 2002; Ruscio, 2010).

For example, I conducted an investigation (O’Brien, 1995) in which eight graduate students who had completed coursework in behavior therapy were provided with a contrived set of self-monitoring data presented on three behaviors: headache frequency, headache intensity, and headache duration. The data set also contained information about four causal variables that could exert a significant influence on headache activity: hours of sleep, marital argument frequency, stress levels, and number of pills taken. The data set was constructed so that each causal variable was strongly correlated with a single aspect of headache activity. Specifically, number of stressors was strongly correlated with headache frequency, $r(13)=.63, p<.05$; number of arguments was strongly correlated with headache severity, $r(13)=.65, p<.05$; hours of sleep was strongly correlated with headache duration , $r(13)=-.77, p<.01$; and number of 
Evaluating Case Formulation Decision-Making and Therapist Responsiveness:

A Perspective from the Area of Behavioral Assessment and Case Formulation

W.H. O'Brien

Pragmatic Case Studies in Psychotherapy, http://pcsp.libraries.rutgers.edu

Volume 6, Module 4, Article 6, pp. 293-306, 12-22-10 [copyright by author]

pills taken was strongly correlated with headache severity, $r(13)=.81, p<.01$. The selfmonitoring data are presented in Table 1.

The graduate students were instructed to evaluate data as they typically would in a clinical setting, estimate the magnitude of correlation between each causal factor and target behavior, and select the most highly associated causal factor for each target behavior. Results indicated that seven of the eight students reported that they used an intuitive evaluation method such as "eyeballing" data. The students also consistently and significantly underestimated the magnitude of large correlations and overestimated the magnitude of weak correlations. Finally, the students were able to correctly identify the most important causal variable for each target behavior less than 50 percent of the time.

We further evaluated the potential limitations of intuitive data evaluation methods by surveying members of a large organization of behavior therapists. Similar to the earlier study, we created a data set that contained three target behaviors and three potential causal variables factors in a $3 \times 3$ table. The correlation between a target behavior and the three causal variables was either low $(\mathrm{r}=.1)$, moderate $(\mathrm{r}=.5)$, or high $(\mathrm{r}=.9)$. Participants were instructed to identify which of the three possible causal variables was most strongly associated with each target behavior. Results indicated that when the true correlation between the target behavior and causal variable was either low or moderate, the participants were able to correctly identify the correct causal variable at slightly better than chance levels. When the true correlation was high, the participants' performance rose to only 72 percent.

Taken together, these results demonstrate that intuitive data evaluation approaches are commonly used by clinicians. However, their ability to reliably and accurately estimate relationships among behaviors and causal factors is very limited. This problem is compounded when one considers the fact that multiple behaviors, multiple causes, and multiple interactions are encountered in a typical assessment.

It is thus recommended that clinicians quantitative decision-making aids, whenever possible, to test the strength of their inferences about behavior- causal factor relationships. A detailed description of these decision making aids is beyond the scope of this commentary. However, many decision-making researchers have argued that clinicians should be well versed in Bayesian statistics (cf., Garb, 2005; Harding, 2007), conditional probability analysis, time series analysis, and quantitative evaluation of single subject data ( Garb, 2005; Haynes, O’Brien, \& Kaholokula, in press).

\section{SUMMARY}

Case formulation is a dynamic and complex decisional process that integrates multiple sources of information. The decision-making processes identified by Eells and Edwards provide insight into important elements of the case formulation and essential clinical competencies. Incorporating additional competencies that were not examined by Eells and Edwards can provide a more comprehensive understanding of the case formulation process. Importantly, it is argued 
Evaluating Case Formulation Decision-Making and Therapist Responsiveness:

A Perspective from the Area of Behavioral Assessment and Case Formulation

W.H. O'Brien

Pragmatic Case Studies in Psychotherapy, http://pcsp.libraries.rutgers.edu

Volume 6, Module 4, Article 6, pp. 293-306, 12-22-10 [copyright by author]

that a core competency is familiarity with the use of quantitative decision-making aids to evaluate the accuracy of intuitive estimates of causal relationships.

\section{REFERENCES}

Chapman, L. J., \& Chapman, J. P. (1969). Illusory correlation as an obstacle to the use of valid psychodiagnostic signs. Journal of Abnormal Psychology, 74, 271-280.

Dollinger, S. J., Greening, L., \& Radtke, R. L. (2001). Reading too much between the lines: Illusory correlation and the word association implications test. Personality and Individual Differences, 30, 799-808.

Edwards, D.J.A. (2010). Using systematic case studies to investigate therapist responsiveness: examples from a case series of PTSD treatments. Pragmatic Case Studies in Psychotherapy, 6(4), Article 3, 255-275. Available: http://hdl.rutgers.edu/1782.1/pcsp_journal

Eells, T. D. (2010). The unfolding case formulation: The interplay of description and inference. Pragmatic Case Studies in Psychotherapy, 6(4), Article 2, 225-254. Available: http://hdl.rutgers.edu/1782.1/pcsp_journal

Garb, H. (2005). Clinical judgment and decision making. Annual Review of Clinical Psychology, 1, 67-89.

Gilovich, T., Griffin, D., \& Kahneman, D. (2002). Heuristics and biases: The psychology of intuitive judgment. New York: Cambridge.

Hayes, S. C., Luoma, J. B., Bond, F. W., Masuda, A., \& Lillis, J. (2006). Acceptance and commitment therapy: Model, processes and outcomes. Behavior Research and Therapy, 44, 1-25.

Haynes, S. N. (1992). Models of causality in psychopathology: Toward synthetic, dynamic, and nonlinear models of causality in psychopathology. Boston: Allyn \& Bacon.

Haynes, S. N., \& O’Brien, W. H. (1990). The functional analysis in behavior therapy. Clinical Psychology Review, 10, 649-668.

Haynes, S. N., \& O’Brien, W. H. (2000). Principles and practice of behavioral assessment. New York: Plenum Press.

Haynes, S. N., O’Brien, W. H., \& Kaholokula, J. K. (in press). Behavioral assessment and case formulation. New York: Wiley.

Huprich, S. (2007). Psychodynamic therapy: Conceptual and empirical foundations. New York: Routledge/Taylor.

Iwata, B. A., Smith, R. G., \& Michael, J. (2000). Current research on the influence of establishing operations on behavior in applied settings. Journal of Applied Behavior Analysis, 33, 411-418.

Nickerson, R. S. (1998). Confirmation bias: A ubiquitous phenomenon in many guises. Review of General Psychology, 2, 175-200.

O’Brien, W. H. (1995). Inaccuracies in the estimation of functional relationships using selfmonitoring data. Journal of Behavior Therapy and Experimental Psychiatry, 26, 351357. 
O’Brien, W. H., \& Haynes, S. N. (1995). A functional analytic approach to the assessment and treatment of a child with frequent migraine headaches. In Session: Psychotherapy in Practice, 1, 65-80.

O’Brien, W. H. Korchynski, R., Fabrizio, J., McGrath, J., \& Swank, A. (1999). Evaluating group process in a stress management intervention: Relationships between perceived process and cardiovascular reactivity to stress. Research on Social Work Practice, 9, 608630.

Tsai, M., Kohlenberg, R., Kanter, J. W., Kohlenberg, B., Follette, W. C., \& Callighan, G. M. (2008). A guide to functional analytic psychotherapy: Awareness, courage, love, and behaviorism. New York: Springer.

Webb, C. A., DeRubreis, R. J. \& Barber, J. P. (2010). Therapist adherence/competencies and treatment outcome: A meta-analysis. Journal of Consulting and Clinical Psychology, 78, 200-211. 
A Perspective from the Area of Behavioral Assessment and Case Formulation

W.H. O'Brien

Pragmatic Case Studies in Psychotherapy, http://pcsp.libraries.rutgers.edu

Volume 6, Module 4, Article 6, pp. 293-306, 12-22-10 [copyright by author]

Table 1.

Headache Activity and Potential Causal Variables

\begin{tabular}{|l|l|l|l|l|l|l|l|}
\hline \multicolumn{9}{|c|}{ Self-Monitoring Information } \\
\hline Day & $\begin{array}{l}\text { Number of } \\
\text { Stressors }\end{array}$ & $\begin{array}{l}\text { Number of } \\
\text { Arguments }\end{array}$ & $\begin{array}{l}\text { Hours } \\
\text { of } \\
\text { Sleep } \\
\text { Frequency }\end{array}$ & $\begin{array}{l}\text { Headache } \\
\text { Feadache } \\
\text { Severity }\end{array}$ & $\begin{array}{l}\text { Headache } \\
\text { Duration } \\
\text { (Hours) }\end{array}$ & $\begin{array}{l}\text { Number } \\
\text { of Pills } \\
\text { Taken }\end{array}$ \\
\hline 1 & 3 & 7 & 8 & 4 & 9 & 1 & 8 \\
\hline 2 & 6 & 1 & 6 & 3 & 5 & 3 & 4 \\
\hline 3 & 5 & 3 & 9 & 6 & 5 & 2 & 4 \\
\hline 4 & 8 & 1 & 5 & 7 & 1 & 4 & 3 \\
\hline 5 & 4 & 2 & 7 & 1 & 6 & 4 & 6 \\
\hline 6 & 7 & 2 & 6 & 5 & 4 & 3 & 4 \\
\hline 7 & 2 & 2 & 8 & 2 & 4 & 1 & 2 \\
\hline 8 & 7 & 5 & 4 & 5 & 7 & 6 & 6 \\
\hline 9 & 2 & 2 & 8 & 3 & 6 & 2 & 4 \\
\hline 10 & 7 & 3 & 5 & 5 & 9 & 3 & 7 \\
\hline 11 & 5 & 3 & 8 & 4 & 6 & 1 & 4 \\
\hline 12 & 3 & 1 & 7 & 5 & 6 & 2 & 4 \\
\hline 13 & 7 & 3 & 7 & 7 & 5 & 1 & 6 \\
\hline 14 & 5 & 3 & 6 & 6 & 8 & 2 & 7 \\
\hline
\end{tabular}

